\title{
Effects of chitosan on dental bone repair
}

\author{
Fatemeh Ezoddini-Ardakani ${ }^{{ }^{*}}$, Alireza Navab Azam ${ }^{2}$, Soghra Yassaei ${ }^{3}$, \\ Farhad Fatehi ${ }^{4}$, Gholamreza Rouhi ${ }^{5}$
}

\footnotetext{
${ }^{1}$ Associate Professor of Oral and Maxillofacial Radiology, Faculty of Dentistry, Shahid Sadoughi University of Medical Sciences, Yazd, Iran; *Corresponding Author: ezoddini@gmail.com;

${ }^{2}$ Lecturer of Oral and Maxillofacial Surgery, Faculty of Dentistry, Shahid Sadoughi University of Medical Sciences, Yazd, Iran;

${ }^{3}$ Associate Professor of Orthodontics, Faculty of Dentistry, Shahid Sadoughi University of Medical Sciences, Yazd, Iran;

${ }^{4}$ Yazd Diabetes Research Center, Shahid Sadoughi University of Medical Sciences, Yazd, Iran;

${ }^{5}$ Department of Mechanical Engineering, Faculty of Engineering \& School of Human Kinetics, Faculty of Health Sciences, University of Ottawa, Ontario, Canada.
}

Received 26 February 2011; revised 12 March 2011; accepted 1 April 2011.

\section{ABSTRACT}

Objectives: Bone defects following tumor resection and osteolysis due to dental and bone lesions and periodentium tissue disorders are serious challenges. One of these materials used is chitosan, a derivative of crustaceans' exoskeleton. The aim of this study was to assess effects of chitosan on socket repair after dental extraction. Methods: Twenty four dental sockets of 15-24 year-old patients were visited by a maxillofacial surgeon for extracting premolar teeth for orthodontic purposes. The sockets in one side were filled-in by chitosan. In the other side, the sockets were left unfilled. After 10 weeks, periapical radiographs were obtained from the repair sites, were digitalized and then evaluated for densitometry using Adobe Photoshop Software. Each socket was divided into coronal, middle and apical. Dental density of each socket in case and control groups was recorded. The density of regenerated bone was compared against the maximum bone density of each individual. Wilcoxon Singed-Rank test and paired t-test were used for data analysis. Results: Bone density in middle and apical sections in case group was significantly more than control group. In apical section in case group regenerated bone reached up to $98.2 \%$ of normal bone density. In each patient, the bone density in epical and middle sections was increase $\mathbf{2 9 . 3 \%}$ and $\mathbf{1 0 . 8 \%}$ of normal bone density. Conclusions: Chitosan significantly increased bone density in epical and middle sections. Chitosan can be used for bone repair in cases of bone loss. Various densitometry studies for evaluating chitosan effects in different bone defects are suggested.

Keywords: Chitosan; Bone Regeneration/Drug Effects; Biocompatible Materials/Administration \& Dosage

\section{INTRODUCTION}

Bone defects may develop in various systemic and dental disorders. Osteolysis in periodontal diseases accounts for the most cases of need for bone repair. The conventional methods of bone repair which commonly are used, such as autografts and allografts have their own shortcomings and drawbacks. Autografts are limited in terms of availability of materials and may result in donor site morbidity [1]. Using allografts may be more desirable in some cases, but the possible immune reaction and infection transmission limit their application. To overcome these limitations, various synthetic bone substitutes made of metal, ceramics, polymers, and various composite structures have been introduced to accelerate and improve the process of bone regeneration; though their safety, effectiveness and efficacy remain uncertain [2]. Recently, by increasing the rate of invasive surgical procedures especially in the fields of orthopedics and dentistry, the bone repair techniques using new materials are getting more popular. The new materials which are used should help us reduce the operation time, scar size, post-operation pain, and also improve patient recovery $[3,4]$. One of the best materials which fulfill these requirements is chitosan [5-7].

Recently a special attention has been made toward using the materials which are derived from nature. Such materials would have some advantages over synthetic ones. Most notably, they have been shown to 
yield faster healing with less incompatibility in human beings [8].

Chitosan is a chitin derived polymer which is produced by deacetylation of chitin. Chitin is mainly found in exoskeleton of crustaceans and also in some fungi. These shells which were simply regarded as garbage in the past times are now seen as a valuable source of chitin [9]. Many biomedical applications have been identified for chitosan including wound healing, bandage, skin grafting, homeostasis, hemodialysis, drug delivery, preventing dental plaque, hypertension control, calcium absorption, bilirubin absorption, and cholesterol control [10-13].

Several desirable properties have been described for chitosan including high osteoinductivity, osteointegratability, easy application and gradual biodegradability that makes it a good candidate for bone regeneration. Some researchers have studied the effects of chitosan compounds on animal bone repair [14-16]. Regarding the characteristics of chitosan as a biomaterial for bone repair, in this study, investigation was made to see the effects of chitosan on dental socket repair after tooth extraction.

\section{MATERIALS AND METHODS}

In this study, we recruited 12 female orthodontic patients with the age of 16 to 24 years old. They were undergoing extraction of 2-4 first premolar teeth as part of their orthodontic treatment and were qualified as ASA class I category. After extraction of the teeth, dental socket on the right jaw was filled with chitosan powder and duly sutured. The cavities on the opposite side got sutured without filling by any excessive material. Chitosan powder was procured from capsules made by Spring Leaf Co., Sydney, Australia. Each capsule contains 250 mg of chitosan powder. The contents of 20 capsules were removed to special plastic bags and sterilized by gamma radiation of 13-15 Kilo gray (KGy).

\subsection{Surgical Procedures}

After anesthetizing the patient by injecting $1 \frac{1}{2}$ cartridge of lidocaine 2\% with 1:100 000 Epinephrine (Darou Pakhsh Pharma. Chem. Co. Tehran, Iran), an intrasulcular incision was made to raise a distal papilla and marginal gingival. This exposed the marginal bone to allow visualization of the alveolar bon level. Extraction of all the first premolars was done at one setting for each patient using a straight elevator and forceps. After extraction of all the premolar teeth, 2cc of fresh blood was collected from the extracted tooth socket and mixed with the chitosan powder for producing a thick pasty material with which each socket on the right side was filled, and the socket on the left side was left unfilled to be used as control. None of the sockets were covered with a barrier membrane or mucoperiosteal flap. The distobuccal, mesiobuccal and palatal papilla with attached gingival at the extraction sites were stabilized with two interrupted suture to reduce the opening of the socket and also the amount of exposed material. All the patients were prescribed a course of prophylactic antibiotic therapy and pain medication with post operative instructions for 7 days, at which point the suture was removed. The dressing of all wounds was performed by the same nurse.

\subsection{Radiological Study}

The patients were recalled 10 weeks after surgery for periapical dental radiography. Periapical radiographs of the extraction sites were obtained using Planmeca Proline X-ray unit (Planmeca Co., Helsinki, Finland) set to $10 \mathrm{KVP}, 8 \mathrm{~mA}$ and $0.16 \mathrm{sec}$. The radiographs were taken by the same technician under the same conditions. The films were processed by Velopex dental x-ray film processors (Medivance Instruments Ltd., London, UK) at $27^{\circ} \mathrm{C}$ for 4 minutes (Figure 1).

\subsection{Qualitative Histopathological Scoring}

The radiographs were digitized by a scanner with 300 DPI resolution and the densitometry was done using Adobe Photoshop software (Adobe Systems Incorporated, San Jose, CA) on a personal computer. Each socket was vertically divided into 3 equal zones: coronal, middle, and apical. Regenerated bone density was assessed

\subsection{Statistical Analyses}

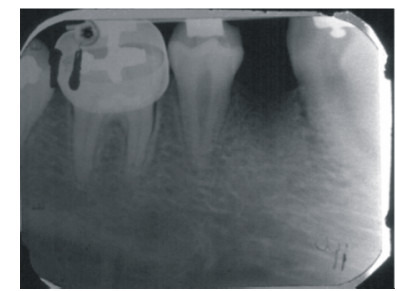

(a)

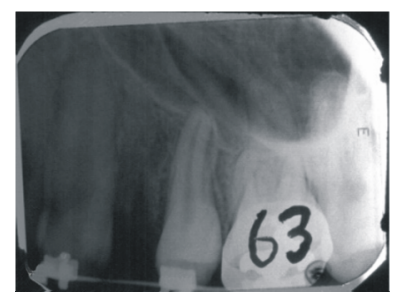

(c)

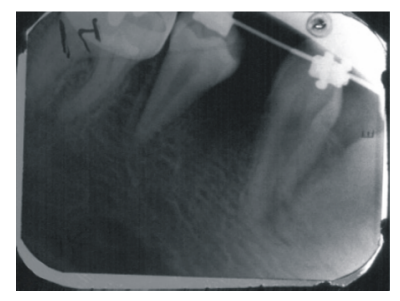

(b)

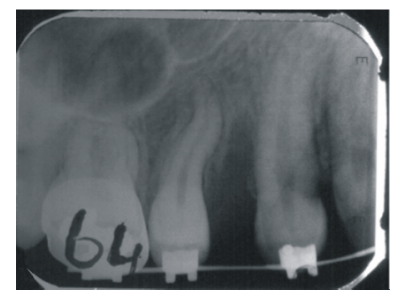

(d)
Figure 1. Periapical radiography of mandibular first premolar in case (a) and control (b); and maxillary first premolar in case (c) and control (d). 
in each zone, in both intervention and control cavities. The density of normal adjacent bone to each cavity was also assessed to be compared with regenerated bone.

Data were analyzed by SPSS ver. 11 (SPSS Inc., Chicago, USA) using Paired t-test and Wilcoxon signed rank test. P-values less than 0.05 were considered as significant.

\subsection{Ethical Consideration}

After explaining the research protocol, an informed consent was obtained from each subject. The proposal of this study got approved by the Ethics Committee of Shahid Sadoughi University of Medical Sciences.

\section{RESULTS}

A total of 24 dental sockets in 12 orthodontics patients were studied. The sockets were either in upper or lower jaws. The sockets on the right side $(n=12)$ were filled with chitosan paste, whereas the sockets on the left $(\mathrm{n}=$ 12) got sutured after tooth extraction without any filling. After ten weeks of tooth extraction, the density of regenerated bone in each socket was assessed for each three zones (coronal, middle, and apical). Extraction site of all the cases healed with no complication. The mean density of the regenerated bone in each zone was assessed by measuring the gray level on the scanned radiographs. The mean density of regenerated bone in each zone of repaired tooth socket for the case and control groups is presented in Table $\mathbf{1}$.

The mean density of regenerated bone was significantly higher in middle and apical zones of case group compared to control group, whereas this difference was not considerable between the coronal zones of the two groups.

As the normal bone density differs from one person to another, the density of regenerated bone in each patient is compared with the maximum bone density of the same patient. The mean bone density of the mandibles of the participants was $92.2 \pm 10.4$ with a range of 78 to 110 . For each person, the ratio of regenerated bone density to the maximum mandibular bone density in both groups of sockets was calculated. These figures and their statistical analysis results and the differences between the two groups are summarized in Table 2.

\section{DISCUSSION}

In this study, an investigated was made on the bone healing effects of chitosan on 12 patients who referred for premolar teeth extraction for orthodontic purposes. The tooth extraction was planned for maxilla, mandible or both of them.

A substance used for improving bone regeneration
Table 1. The mean gray level of each zone of the sockets in case and control subjects.

\begin{tabular}{llll}
\hline Group & $\begin{array}{l}\text { Coronal } \\
\text { mean } \pm \text { SD } \\
(\text { min } \sim \text { Max })\end{array}$ & $\begin{array}{l}\text { Middle } \\
\text { mean } \pm \text { SD } \\
(\text { min } \sim \text { Max })\end{array}$ & $\begin{array}{l}\text { Apical } \\
\text { mean } \pm \text { SD } \\
(\text { min } \sim \text { Max })\end{array}$ \\
\hline $\begin{array}{l}\text { Chitosan } \\
(\mathrm{n}=12)\end{array}$ & $\begin{array}{l}35.3 \pm 12.0 \\
(21.7 \sim 60)\end{array}$ & $\begin{array}{l}57.7 \pm 12.3 \\
(42 \sim 78.2)\end{array}$ & $\begin{array}{l}90.9 \pm 12.5 \\
(68 \sim 110)\end{array}$ \\
$\begin{array}{l}\text { Control } \\
(\mathrm{n}=12)\end{array}$ & $\begin{array}{l}34.2 \pm 1.6 \\
(22.8 \sim 57.1)\end{array}$ & $\begin{array}{l}47.3 \pm 13.4 \\
(31.7 \sim 76.3)\end{array}$ & $\begin{array}{l}64 \pm 16.5 \\
(34.3 \sim 95.5)\end{array}$ \\
P value* $^{*}$ & 0.583 & 0.05 & 0.002 \\
\hline
\end{tabular}

*Wilcoxon Signed Rank Test.

Table 2. Ratio of regenerated bone density to the maximum mandibular bone density at the three zones in chitosan-filled and control groups.

\begin{tabular}{llll}
\hline Group & $\begin{array}{l}\text { Coronal } \\
\% \pm S D\end{array}$ & $\begin{array}{l}\text { Middle } \\
\% \pm S D\end{array}$ & $\begin{array}{l}\text { Apical } \\
\% \pm S D\end{array}$ \\
\hline Chitosan $(\mathrm{n}=12)$ & $37.8 \pm 9.8$ & $61.9 \pm 7.9$ & $98.2 \pm 3.9$ \\
Control $(\mathrm{n}=12)$ & $37.3 \pm 11.6$ & $51.1 \pm 13.1$ & $68.9 \pm 14.6$ \\
P value * & 0.896 & 0.040 & 0.000 \\
\hline
\end{tabular}

* Paired t-test.

should be biocompatible, biodegradable, and effective. It should also be cheap and easy to apply [1]. The gold standard for restoring missing bone is autogenous bone graft, which is hard to perform and has some limitationsand drawbacks [17-19]. Moreover, this method is associated with the risk of graft rejection and/or immunological reactions $[9,10]$. Chitosan has been reported as a biodegradable and biocompatible substance [20], and according to numerous studies it is effective in restoring bone defects [21-26]. Chitosan can be used as a biocompatible coating for orthopedic and craniofacial implants [14]. Minimal inflammatory reactions have been observed in tissues which have been in contact with the chitosan coated pins; while the healing sequence of bone remains typical. So, chitosan coatings have shown to be able to develop suitable osseointegration of dental and orthopedic implants [27]. Chitosan microparticles can also improve drug delivery to localized areas which leads to increased and accelerated bone growth [28].

Several studies have investigated various effects of chitosan on bone healing and raised some hypotheses on its mechanisms [29-31]. For instance, according to a study by Chevrier and co-workers, chitosan increases the vascularization of blood vessels and stimulates budding tissue (tissue comprising of budding capillaries and fibroblasts) [32].

Park and co-workers [33] reported that spongy chitosan activates osteoblasts and could increase osteogenesis. 
Klokkevold [34] also reported that chitosan increases the activity of osteoblasts and helps bone formation. Lee and co-workers [35] reported that spongy chitosan supports the proliferation of osteoblastic cells. Considering the rate of bone formation and the speed of bone regeneration in the dental cavities (see Tables $\mathbf{1}$ and 2), results of this study are in agreement of the above mentioned studies.

Kim and co-workers [36] studied chitosan and its derivatives and their applications in tissue engineering, such as the formation of skin, bone, cartilage, liver, nerves, and blood vessels. In the present study, chitosan powder is used to see its effect on bone regeneration. It was interestingly found that after a period of 10 weeks, the bone density in the apical zone of the sockets treated with chitosan was $98.2 \%$ of maximum mandibular bone density, which was $29.3 \%$ more than that of untreated sockets.

In a study by Zhang and co-workers [37], chitosan was used as a biocompatible and biodegradable polymer along with mannitol and calcium phosphate cement (CPC) for bone healing. They reported that this new formulation could be used for shaping hydroxyapatite in surgeries and implants. This new formulation can be used in improving the macroporosity of apatitie frameworks, in order to help reduce the stress shielding in an implant-bone complex, also implant longetivity. In our study, higher speed of bone formation in the apical and middle zones of the dental sockets filled with chitosan can be justified by an increase in scaffold and positioning of the bone forming cells in this framework. $\mathrm{Xu}$ and co-workers [38] used CPC for repair of teeth and craniofacial tissue. In their study, CPC was used for repair of periodontal bony tissue and loose teeth following fractures. They used tetra-calcium phosphate and chitosan in order to make non-rigid and strong calcium phosphate cement, which they believe is more useful in repair of periodontal tissue and bone surrounding an implant. Chitosan has been used also for producing fast-setting CPC and makes it resistant to washout [38]. Chitosan can solve the problem of handling the particulate form of calcium hydroxyapatite as it can stabilize the particles in surgical sites [39]. Bumgardner and co-workers [14] reported that chitosan is a biopolymer that accelerates bone formation, facilitates wound healing and has antimicrobial properties. It also helps bone formation and makes orthopedic procedures and craniofacial implants easier. In the present study, the chitosan powder was mixed with blood of each person and filled in the dental socket, and it was found that bone tissue regeneration will be faster in chitosan-filled socket than untreated dental socket.

Ma and co-workers [40] studied the heat sensitive effects of chitosan hydrogel on periodontal bone healing.
They concluded that chitosan thermosensitive hydrogel loading rhBMP-2 can facilitate regeneration of the periodontal tissue and simplify the surgical operation. Defects were made in the anterior section of the jaws of three healthy dogs and chitosan hydrogel was injected in the wounds and the flaps were sutured. But, a number of defects were left untreated and not filled with the hydrogel. After a period of 5 weeks, the periodontal tissue was regenerated in all main regions of the study group, while only a small section of the tissue was regenerated in the control group. In their study, in the cavities filled with chitosan, not only the bone regeneration was faster, but also the density was similar to the density of the bone of the subject under study. Zhang and co-workers [41] used chitosan scaffold and adenovirus vector for regeneration of alveolar bone in dental implant defects. They reported that chitosan-collagen scaffold can be used as a good mediator in bone regeneration.

\section{CONCLUSION}

Chitosan has been shown to be one of the most promising biomaterials for orthopedic and dental applications. Due to its interesting characteristics, chitosan is considered as a suitable alternative for bone graft. Chitosan improves bone regeneration in dental bone loss.

\section{REFERENCES}

[1] Wang, X., Ma, J., Wang, Y. and He, B. (2002) Bone repair in radii and tibias of rabbits with phosphorylated chitosan reinforced calcium phosphate cements. Biomaterials, 23, 4167-4176.

doi:10.1016/S0142-9612(02)00153-9

[2] Damien, C.J. and Parsons, J.R. (1991) Bone graft and bone graft sub-stitutes: A review of current technology and applications. Journal of Applied Biomaterials, 2, 187-208. doi:10.1002/jab.770020307

[3] Liu, H., Li, H., Cheng, W., Yang, Y., Zhu, M. and Zhou, C. (2006) Novel injectable calcium phosphate/chitosan composites for bone substitute materials. Acta Biomater, 2, 557-565. doi:10.1016/j.actbio.2006.03.007

[4] Song, H.Y., Esfakur Rahman, A.H. and Lee, B.T. (2009) Fabrication of calcium phosphate-calcium sulfate injectable bone substitute using chitosan and citric acid. Journal of Materials Science: Materials in Medicine, 20, 935-941. doi:10.1007/s10856-008-3642-8

[5] Khan, T.A., Peh, K.K. and Ch’ng, H.S. (2002) Reporting degree of dea-cetylation values of chitosan: The influence of analytical methods. Journal of Pharmacy \& Phar-maceutical Sciences, 5, 205-212.

[6] Madihally, S.V. and Matthew, H.W. (1999) Porous chitosan scaffolds for tissue engineering. Biomaterials, 20, 1133-1142. doi:10.1016/S0142-9612(99)00011-3

[7] Nascimento, E.G., Sampaio, T.B., Medeiros, A.C. and Azevedo, E.P. (2009) Evaluation of chitosan gel with 1\% silver sulfa-diazine as an alternative for burn wound 
treatment in rats. Acta Cirurgica Brasileira, 24, 460-465. doi:10.1590/S0102-86502009000600007

[8] Khor, E. and Lim, L.Y. (2003) Implantable applications of chitin and chitosan. Biomaterials, 24, 2339-249. doi:10.1016/S0142-9612(03)00026-7

[9] Spin-Neto, R., de Freitas, R.M., Pavone, C., Cardoso, M.B., Campana-Filho, S.P., Marcantonio, R.A., et al. (2010) Histological evaluation of chitosan-based biomaterials used for the correction of critical size defects in rat's calvaria. Journal of Biomedical Materials Research, 93, 107-114.

[10] Costantino, P.D., Friedman, C.D. and Lane, A. (1993) Synthetic bioma-terials in facial plastic and reconstructive surgery. Facial Plastic Surgery, 9, 1-15. doi:10.1055/s-2008-1064591

[11] LeHoux, J.G. and Grondin, F. (1993) Some effects of chitosan on liver function in the rat. Endocrinology, 132, 1078-1084. doi:10.1210/en.132.3.1078

[12] Machida, Y., Nagai, T., Abe, M. and Sannan, T. (1986) Use of chitosan and hydroxypropylchitosan in drug formulations to effect sustained release. Drug Design \& Delivery, 1, 119-130.

[13] Muzzarelli, R.A., Biagini, G., Bellardini, M., Simonelli, L., Castaldini, C. and Fratto, G. (1993) Osteoconduction exerted by methylpyrrolidinone chitosan used in dental surgery. Biomaterials, 14, 39-43. doi:10.1016/0142-9612(93)90073-B

[14] Bumgardner, J.D., Wiser, R., Gerard, P.D., Bergin, P., Chest-nutt, B., Marin, M., et al. (2003) Chitosan: Potential use as a bioactive coating for orthopaedic and craniofacial/dental implants. Journal of Biomaterials Science, Polymer Edition, 14, 423-438. doi:10.1163/156856203766652048

[15] Ducy, P., Schinke, T. and Karsenty, G. (2000) The osteoblast: A sophisticated fibroblast under central surveillance. Science, 289, 1501-1504. doi:10.1126/science.289.5484.1501

[16] Yao, Z., Xing, L., Qin, C., Schwarz, E.M. and Boyce, B.F. (2008) Osteoclast precursor interaction with bone matrix induces osteoclast formation directly by an interleukin-1-mediated autocrine mechanism. Journal of Biological Chemistry, 283, 9917-9924. doi:10.1074/jbc.M706415200

[17] Karesh, J.W. (1998) Biomaterials in ophthalmic plastic and reconstructive surgery. Current Opinion in Ophthalmology, 9, 66-74. doi:10.1097/00055735-199810000-00013

[18] Miyamoto, Y., Ishikawa, K., Takechi, M., Toh, T., Yuasa, T., Nagayama, M., et al. (1998) Basic properties of calcium phosphate cement containing atelocollagen in its liquid or powder phases. Biomaterials, 19, 707-715. doi:10.1016/S0142-9612(97)00186-5

[19] Nguyen, H., Qian, J.J., Bhatnagar, R.S. and Li, S. (2003) Enhanced cell attachment and osteoblastic activity by P-15 peptide-coated matrix in hydrogels. Biochemical Biophysical Research Communications, 311, 179-186. doi:10.1016/j.bbrc.2003.09.192

[20] Jayakumar, R., New, N., Tokura, S. and Tamura, H. (2007) Sulfated chitin and chitosan as novel biomaterials. International Journal of Biological Macromolecules, 40, 175-181. doi:10.1016/j.ijbiomac.2006.06.021

[21] Cui, X., Zhang, B., Wang, Y. and Gao, Y. (2008) Effects of chitosan-coated pressed calcium sulfate pellet combined with recombinant human bone morphogenetic protein 2 on restoration of segmental bone defect. Journal of Craniofacial Surgery, 19, 459-465. doi:10.1097/SCS.0b013e31815ca034

[22] Hirano, S. and Noishiki, Y. (1985) The blood compatibility of chitosan and N-acylchitosans. Journal of Biomedical Materials Research, 19, 413-417. doi:10.1002/jbm.820190406

[23] Lee, K.Y., Ha, W.S. and Park, W.H. (1995) Blood compatibility and biodegradability of partially N-acylated chitosan derivatives. Biomaterials, 16, 1211-1216. doi:10.1016/0142-9612(95)98126-Y

[24] VandeVord, P.J., Matthew, H.W., DeSilva, S.P., Mayton, L., Wu, B. and Wooley, P.H. (2002) Evaluation of the biocompatibility of a chitosan scaffold in mice. Journal of Biomedical Materials Research, 59, 585-590. doi:10.1002/jbm.1270

[25] Xu, C.J., Guo, F., Gao, Q.P., Wu, Y.F., Jian, X.C. and Peng, J.Y. (2006) Effects of astragalus polysaccharides -chitosan/polylactic acid scaffolds and bone marrow stem cells on repairing supra-alveolar periodontal defects in dogs. ZhongNanDaXueXueBaoYiXueBan, 31, 512-517.

[26] Yeo, Y.J., Jeon, D.W., Kim, C.S., Choi, S.H., Cho, K.S., Lee, Y.K., et al. (2005) Effects of chitosan nonwoven membrane on periodontal healing of surgically created one-wall intrabony defects in beagle dogs. Journal of Biomedical Materials Research, Part B: Applied Biomaterials, 72, 86-93. doi:10.1002/jbm.b.30121

[27] Bumgardner, J.D., Chesnutt, B.M., Yuan, Y., Yang, Y., Appleford, M., Oh, S., et al. (2007) The integration of chitosan-coated titanium in bone: An in vivo study in rabbits. Implant Dentistry, 16, 66-79.

[28] Jayasuriya, A.C. and Kibbe, S. (2010) Rapid biomineralization of chitosan microparticles to apply in bone regeneration. Journal of Materials Science: Materials in Medicine, 21, 393-398. doi:10.1007/s10856-009-3874-2

[29] Mizuno, K., Yamamura, K., Yano, K., Osada, T., Saeki, S., Takimoto, N., et al. (2003) Effect of chitosan film containing basic fibroblast growth factor on wound healing in genetically diabetic mice. Journal of Biomedical Materials Research, Part A, 64, 177-181. doi:10.1002/jbm.a.10396

[30] Ueno, H., Murakami, M., Okumura, M., Kadosawa, T., Uede, T. and Fujinaga, T. (2001) Chitosan accelerates the production of osteopontin from polymorphonuclear leukocytes. Biomaterials, 22, 1667-1673. doi:10.1016/S0142-9612(00)00328-8

[31] Ueno, H., Nakamura, F., Murakami, M., Okumura, M., Kadosawa, T. and Fujinag, T. (2001) Evaluation effects of chitosan for the extracellular matrix production by fibroblasts and the growth factors production by macrophages. Biomaterials, 22, 2125-2130. doi:10.1016/S0142-9612(00)00401-4

[32] Chevrier, A., Hoemann, C.D., Sun, J. and Buschmann, M.D. (2007) Chitosanglycerol phosphate/blood implants increase cell recruitment, transient vascularization and subchondral bone remodeling in drilled cartilage defects. Osteoarthritis Cartilage, 15, 316-327. doi:10.1016/j.joca.2006.08.007

[33] Park, Y.J., Lee, Y.M., Park, S.N., Sheen, S.Y., Chung, C.P. and Lee, S.J. (2000) Platelet derived growth factor 
releasing chitosan sponge for periodontal bone regeneration. Biomaterials, 21, 153-159.

doi:10.1016/S0142-9612(99)00143-X

[34] Klokkevold, P.R. and Newman, M.G. (2000) Current status of dental implants: A periodontal perspective. International Journal of Oral \& Maxillofacial Implants, 15, 56-65.

[35] Lee, Y.M., Park, Y.J., Lee, S.J., Ku, Y., Han, S.B., Choi, S.M., et al. (2000) Tissue engineered bone formation using chitosan/tricalcium phosphate sponges. Journal of Periodontology, 71, 410-417. doi:10.1902/jop.2000.71.3.410

[36] Kim, I.Y., Seo, S.J., Moon, H.S., Yoo, M.K., Park, I.Y., Kim, B.C., et al. (2008) Chitosan and its derivatives for tissue engineering applications. Biotechnology Advances, 26, 1-21. doi:10.1016/j.biotechadv.2007.07.009

[37] Zhang Y., Xu, H.H., Takagi, S. and Chow, L.C. (2006) In-situ hardening hydroxyapatite-based scaffold for bone repair. Journal of Materials Science: Materials in Medicine, 17, 437-445. doi:10.1007/s10856-006-8471-z

[38] Xu, H.H., Takagi, S., Quinn, J.B. and Chow, L.C. (2004)
Fast-setting calcium phosphate scaffolds with tailored macropore formation rates for bone regeneration. Journal of Biomedical Materaials Research: Part A, 68, 725-734. doi:10.1002/jbm.a.20093

[39] Pal, A.K., Pal, T.K., Mukherjee, K. and Pal, S. (1997) Animal experimentation with tooth derived calcium hydroxyapatite based composites as bone-graft substitute biomaterials. Biomedical Sciences Instrumentation, 33, 561-566.

[40] Ma, Z.W,. Zhang, Y.J., Wu, Z.F., Wang, R., Zhu, H., Li, Y., et al. (2008) A study on the effect of the chitosan thermosensitive hydrogel loading recombinant human bone morphogenetic protein-2 on repairing periodontal defects. Hua XiKou QiangYiXueZa Zhi, 26, 23-26.

[41] Zhang, Y., Song, J., Shi, B., Wang, Y., Chen, X., Huang, C., et al. (2007) Combination of scaffold and adenovirus vectors expressing bone morphogenetic protein-7 for alveolar bone regeneration at dental implant defects. Biomaterials, 28, 4635-4642. doi:10.1016/j.biomaterials.2007.07.009 